\title{
Active Sampling for Knowledge Discovery from Biomedical Data
}

\author{
Sriharsha Veeramachaneni ${ }^{1}$, Francesca Demichelis ${ }^{1,2}$, \\ Emanuele Olivetti ${ }^{1}$, and Paolo Avesani ${ }^{1}$ \\ 1 SRA Division, ITC-IRST, Trento, Italy 38050 \\ 2 Department of Pathology, Brigham and Women's Hospital, \\ Harvard Medical School, Boston, USA \\ \{sriharsha, michelis, olivetti, avesani\}@itc.it
}

\begin{abstract}
We describe work aimed at cost-constrained knowledge discovery in the biomedical domain. To improve the diagnostic/prognostic models of cancer, new biomarkers are studied by researchers that might provide predictive information. Biological samples from monitored patients are selected and analyzed for determining the predictive power of the biomarker. During the process of biomarker evaluation, portions of the samples are consumed, limiting the number of measurements that can be performed. The biological samples obtained from carefully monitored patients, that are well annotated with pathological information, are a valuable resource that must be conserved. We present an active sampling algorithm derived from statistical first principles to incrementally choose the samples that are most informative in estimating the efficacy of the candidate biomarker. We provide empirical evidence on real biomedical data that our active sampling algorithm requires significantly fewer samples than random sampling to ascertain the efficacy of the new biomarker.
\end{abstract}

\section{Introduction}

In the biomedical domain, the acquisition of data is often expensive. The cost constraints generally limit the amount of data that is available for analysis and knowledge discovery. We present a methodology for intelligent incremental data acquisition for performing knowledge discovery under cost constraints.

In biological domains molecular tests, called biomarkers, conducted on biological samples (e.g. tumor tissue samples) that provides predictive information to pre-existing clinical data are studied. The development of molecular biomarkers for clinical application is a long process that must go through many phases starting from early discovery phases to more formalized clinical trials. This process involves the analysis of biological samples obtained from a large population of patients in a retrospective manner. The biological samples that need to be properly preserved are collected together with corresponding clinical data over time and are therefore very valuable. There is therefore a need to carefully optimize the use of the samples while studying new biomarkers. We address the issue 
of cost-constrained biomarker evaluation for developing diagnostic/prognostic models for cancer.

In general the acquisition of new data can be performed automatically by querying the environment by choosing the queries that are most likely to provide 'useful' information. This learning paradigm called active learning, where the learner is endowed with the ability to choose the data to be acquired, has been shown to yield comparable accuracy with significantly fewer data 16911. Traditionally active learning methods have been applied for training classifiers in the presence of unlabeled data, where the class labels of carefully chosen samples are queried. These techniques are suitable for situations where the class labels are considerably more expensive to obtain than the feature representation of the examples. Moreover the queries are chosen in order to learn the classifier accurately (with low cost).

In contrast, for our problem new biomarkers are tested on biological samples from patients who are labeled according to their disease and survival status. Moreover for each patient we have additional information such as grade of the disease, tumor dimensions and lymphonode status. That is, the samples are class labeled as well as described by some previous features. The goal is to choose the new feature (the biomarker) among many that is most correlated with the class label given the previous features. Since the cost involved in the evaluation of all the biomarkers on all the available data is prohibitive, we need to actively choose the the samples on which the new features (biomarkers) are tested. Therefore our objective at every sampling step is to choose the query (the sample on which the new feature is measured) so as to learn the efficacy of the biomarker most accurately.

Although the general theory of active learning has been studied in statistics in the area of optimal experimentation [71013], it has seldom been applied to problems in knowledge discovery. The reason being the difficulty in resolving various practical issues such as finding good approximations to the theory and learning with sampling bias (which is a side-effect of active sampling).

In Section 2 we provide an overview of the process of identifying and evaluating biomarkers with a description of the resources required. In Section 3 we present an abstract formulation of the problem and outline a solution. We then describe the dataset obtained from Tissue Microarray analysis and will provide experimental evidence for the efficacy of our solution. We conclude with a discussion of the insights gained and directions for future work.

\section{Cancer Biomarker Evaluation}

Current models for cancer characterization, that lead to diagnostic/prognostic models, mainly involve the histological parameters (such as grade of the disease, tumor dimensions, lymphonode status) and biochemical parameters (such as the estrogen receptor). The diagnostic models used for clinical cancer care are not 
yet definitive and the prognostic and therapy response models do not accurately predict patient outcome and follow up. For example, for lung cancer, individuals affected by the same disease and equally treated demonstrate different treatment responses, evidencing that still unknown tumor subclasses (different istotypes) exist. This incomplete view results, at times, in the unnecessary over-treatment of patients, that is some patients do not benefit from the treatment they undertake. Diagnostic and prognostic models used in clinical cancer care can be improved by embedding new biomedical knowledge. The ultimate goal is to improve the diagnostic and prognostic ability of the pathologists and clinicians leading to better decisions about treatment and care.

Ongoing research in the study and characterization of cancer is aimed at the refinement of the current diagnostic and prognostic models. As disease development and progression are governed by gene and protein behaviour, new biomarkers found to be associated with patient diagnosis or prognosis are investigated. The identification of new potential biomarkers is recently driven by high throughput technologies, called Microarrays. They enable the identification of genes that provide information with a potential impact on understanding disease development and progression [5].

Although the initial high throughput discovery techniques are rapid, they often only provide qualitative data. Promising genes are further analyzed by using other experimental approaches (focusing on DNA, RNA or proteins), to test specific hypotheses. Usually a well characterized dataset of tumor samples from a retrospective population of patients is identified and the experimental process of analyzing one biomarker (feature) on one sample at a time is conducted. These analyses are usually based on the comparison between 1)non-diseased (e.g. normal) and diseased (e.g. tumors) biological samples, 2)between diseased samples pharmacologically treated and untreated at variable time points or 3)between samples of different diseases. The efficacy of specific biomakers can for example be determined based on their discriminative power in distinguishing between patients with poor or good prognosis, meaning patients with short or long overall survival respectively or cancer recurring or not recurring. This process can be time consuming, depending on the type of experimental technique which is adopted.

More importantly, well annotated tissue samples are very precious. Monitoring the status of patients over years, even decades, and store them so that to be useful for studies is not trivial and requires organizational efforts. It is not uncommon, for example, that patients who undertook the treatment at a hospital, will be monitored during the follow up period in another hospital and even in another country. Therefore keeping track of their status may become quite difficult. When the biomarker is tested on a biological sample, a portion of the sample is consumed, implying that each sample can be used for only a finite number of experiments. This motivates the need to develop an active sampling approach to conserve the valuable biological sample resource. 


\section{Active Measurement of Feature Values}

We first present an abstract statement of the problem and show how it fits the real problem in the biomedical domain. We will then derive an active sampling algorithm as a solution. Let us consider a finite set of monitored pattern instances (or subjects) $T=\left\{t_{i}\right\}_{i=1, \ldots, N}$. Let the random variable corresponding to the class label be denoted by $\mathbf{c}$ taking values in $\mathcal{C}$. The random variables $1 \mathbf{x}$ and $\mathbf{y}$ correspond to features (or attributes) that can be measured on any instance, taking on values in $\mathcal{X}$ and $\mathcal{Y}$ respectively. The class label and the feature value $\mathbf{x}$ are known for every instance in $T$. In other words the random vector $\mathbf{s}=(\mathbf{c}, \mathbf{x})$ is instantiated on all the instances. However, initially, feature value $\mathbf{y}$ has not been acquired on any instance. Let the probability distribution over $\mathcal{C} \times \mathcal{X} \times \mathcal{Y}$ be parameterized by $\theta \in \Theta$. It is required to learn a concept $g$ (which is a function of $\theta$ ) on $\mathcal{C} \times \mathcal{X} \times \mathcal{Y}$ accurately by minimizing the number of instances on which $\mathbf{y}$ is measured (or probed).

In this formulation the class label $\mathbf{c}$ represents the diagnostic class, overall survival or relapse free survival status or the response to the treatment of the particular patient, the previous feature vector $\mathbf{x}$ represents the histological parameters of the tumor sample or biomarkers previously determined to be effective and the feature $\mathbf{y}$ represents the expression level of the candidate biomarker that is being evaluated. The concept $\mathbf{g}$ to be learned represents the efficacy of the new feature, or equivalently the error rate (denoted by e) of the classifier $\phi: \mathcal{X} \times \mathcal{Y} \rightarrow \mathcal{C}$ operating on the full feature space. Since the goal is to save the valuable resource we would like to measure as few samples as possible with the new biomarker before deciding whether it provides any useful information.

\subsection{Active Sampling to Minimize Predicted Mean Squared Error}

The active sampling approach is to iteratively choose the best $s=(c, x)$ where to probe the feature value $\mathbf{y}$. Then an instance in the dataset with the particular $s$ is chosen on which $\mathbf{y}$ is probed. Let us assume that after $k$ iterations of this sampling process we obtained the dataset of measurements $T_{k}=\left\{s_{1} \rightarrow y_{1}, s_{2} \rightarrow\right.$ $\left.y_{2}, \ldots, s_{k} \rightarrow y_{k}\right\}$. We will now describe how the choice of best $s$ can be performed from a Bayesian statistical viewpoint.

Let us assume that the estimate of the concept is the Bayes minimum mean square error (MMSE) estimate. That is, given data $T$, the estimate of the concept is given by $\hat{g}(T)=E[\mathbf{g} \mid T]$. The predicted mean squared error (MSE) of the estimate of $g$ at step $k+1$, if $s$ were to be probed for the value of $\mathbf{y}$, is given by

$$
\begin{aligned}
\operatorname{MSE}(s)_{k+1} & =\iint\left(E\left[\mathbf{g} \mid T_{k}, s \rightarrow y\right]-g\right)^{2} p\left(g \mid T_{k}, s \rightarrow y\right) p\left(s \rightarrow y \mid T_{k}\right) d g d y \\
& =\iint\left(E\left[\mathbf{g} \mid T_{k}, s \rightarrow y\right]-g\right)^{2} p\left(g, s \rightarrow y \mid T_{k}\right) d g d y
\end{aligned}
$$

\footnotetext{
$\overline{{ }^{1} \text { In general } \mathbf{x}}$ and $\mathbf{y}$ can be random feature vectors of different lengths.
} 
Note that the MSE is averaged over all the possible values of $\mathbf{y}$, with the probabilities given by conditioning over all the data we have seen thus far. Now the best $s$ to probe is the one that yields the lowest predicted mean squared error 2 .

Lemma 1. To select the sample s to minimize the predicted mean squared error in Equation 1, we can equivalently maximize the squared difference between the Bayes estimates of the concept before and and after $s$ is probed, averaged over the possible outcomes. That is

$$
\begin{aligned}
B(s) & =\underset{s}{\operatorname{argmin}} \operatorname{MSE}(s)_{k+1} \\
& =\underset{s}{\operatorname{argmax}} \int_{\mathcal{Y}}\left(E\left[\mathbf{g} \mid T_{k}, s \rightarrow y\right]-E\left[\mathbf{g} \mid T_{k}\right]\right)^{2} p\left(s \rightarrow y \mid T_{k}\right) d y
\end{aligned}
$$

The proof is outlined in [12. The result implies that the most informative $s$ to sample at is the one where the sampling would lead to the most change from the current estimate of the concept in the expected sense. In most problems, however, it is difficult to estimate the concept using a Bayes MMSE approach. Therefore we relax this constraint to approximate the objective function as

$$
B(s)=\int_{\mathcal{Y}}\left(\hat{g}\left(T_{k}, s \rightarrow y\right)-\hat{g}\left(T_{k}\right)\right)^{2} p\left(s \rightarrow y \mid T_{k}\right) d y
$$

where $\hat{g}(T)$ is any estimate of the concept $\mathbf{g}$ from data $T$, that is appropriate for the problem. Our active sampling method based on this benefit criterion is called the Maximum Average Change (MAC) sampling algorithm 3 . An approach such as ours where the best instance to sample is decided based upon the benefit at the next step is called myopic active learning. An alternative would be reason about the best sample based upon the benefit it will have after many sampling steps. This alternative, however, can be computationally very expensive.

For feature selection we need to evaluate the benefit of adding each candidate feature $\mathbf{y}$ to the current set of features $\mathbf{x}$. Therefore the concept $\mathbf{g}$ to be learned is the error rate (denoted by $\mathbf{e}$ ) of the classifier $\phi: \mathcal{X} \times \mathcal{Y} \rightarrow \mathcal{C}$ operating on the full feature space. Although the above derivation of the active learning strategy is problem and classifier independent, we implemented the active feature measurement algorithm for the estimation of the error rate of a Bayesian maximum a posteriori classifier with discrete valued features and class labels. As opposed to the Naive Bayes classifier, we do not assume that the features $\mathbf{x}$ and $\mathbf{y}$ are class-conditionally independent. We will now briefly describe how the active sampling algorithm was implemented and provide the equations for the estimation of the probability distribution and for the error rate of the classifier.

${ }^{2}$ In case of non-uniform costs a different objective function that uses both the sampling cost and the MSE should be optimized.

${ }^{3}$ In 12 we have shown that MAC heuristic incurs significantly lower sampling cost than the heuristics proposed by Lizotte et al. 4 and Zheng and Padmanabhan 14 for similar problems. 


\subsection{Implementation}

All probability distributions are multinomial whose parameters are estimated from data using Bayes MMSE estimators under uniform Dirichlet priors. Due to the difficulty in obtaining the exact Bayes MMSE estimate of the error rate, we approximate it by the error rate computed from the Bayes estimate of the distribution $p(c, x, y)$ over $\mathcal{C} \times \mathcal{X} \times \mathcal{Y}$.

We will now describe how the estimation of the joint probability is performed and present the formulae for the computation of the classifier and its error rate. At a given iteration of the active sampling process some of the instances have feature value $\mathbf{y}$ missing. Moreover because of the active sampling the missing values are not uniformly distributed. In [3] MacKay asserts that the biases introduced in the induced concept because of non-random sampling can be avoided by taking into account how we gathered the data. Therefore to construct the estimator $\hat{p}(c, x, y)$ over $\mathcal{C} \times \mathcal{X} \times \mathcal{Y}$ it is necessary to consider the sampling process. Since all the examples in the database are completely described with respect to $\mathbf{c}$ and $\mathbf{x}$ we already have the density $p(c, x)$. In addition, at any iteration of the active sampling algorithm there is an incomplete database with $\mathbf{y}$ values missing non-uniformly across various configurations of $(\mathbf{c}, \mathbf{x})$. However for each $(c, x)$ the samples for $y$ are independent and identically distributed. We incorporate this information in the estimator of the probability density from incomplete data $T$ as follows. We first calculate

$$
\hat{p}_{T}(y \mid c, x)=\frac{n_{c, x, y}+1}{\sum_{\mathcal{Y}} n_{c, x, y}+|\mathcal{Y}|}
$$

where $n_{c, x, y}$ is the number of instances of the particular combination of $(c, x, y)$ among all the completely described instances in $T$. Note that $\hat{p}_{T}(y \mid c, x)$ is the same as $p(s \rightarrow y \mid T)$ used in the equations above. Now the probability density over $\mathcal{C} \times \mathcal{X} \times \mathcal{Y}$ is calculated as

$$
\hat{p}_{T}(c, x, y)=\hat{p}_{T}(y \mid c, x) \times p(c, x)
$$

Once we have the estimate $\hat{p}_{T}(c, x, y)$ all other quantities can be computed easily and in particular the estimate of the error rate $\hat{e}(T)$ is computed as follows.

$$
\hat{e}(T)=1-\sum_{\mathcal{X} \times \mathcal{Y}} \hat{p}_{T}(\hat{\phi}(x, y), x, y)
$$

where $\hat{\phi}_{T}$ is the Bayes maximum a posteriori classifier learned from data $T$ given by

$$
\hat{\phi}_{T}(x, y)=\underset{c \in \mathcal{C}}{\operatorname{argmax}} \frac{\hat{p}_{T}(c, x, y)}{\sum_{\mathcal{C}} \hat{p}_{T}(c, x, y)}
$$

For a given amount of budget and candidate feature $\mathbf{y}$, the MAC active sampling algorithm to learn the utility of (i.e., the error rate given) the feature is given in pseudocode below. 
Algorithm : ActiveSamplingForErrorRate(DataSet,y, Budget)

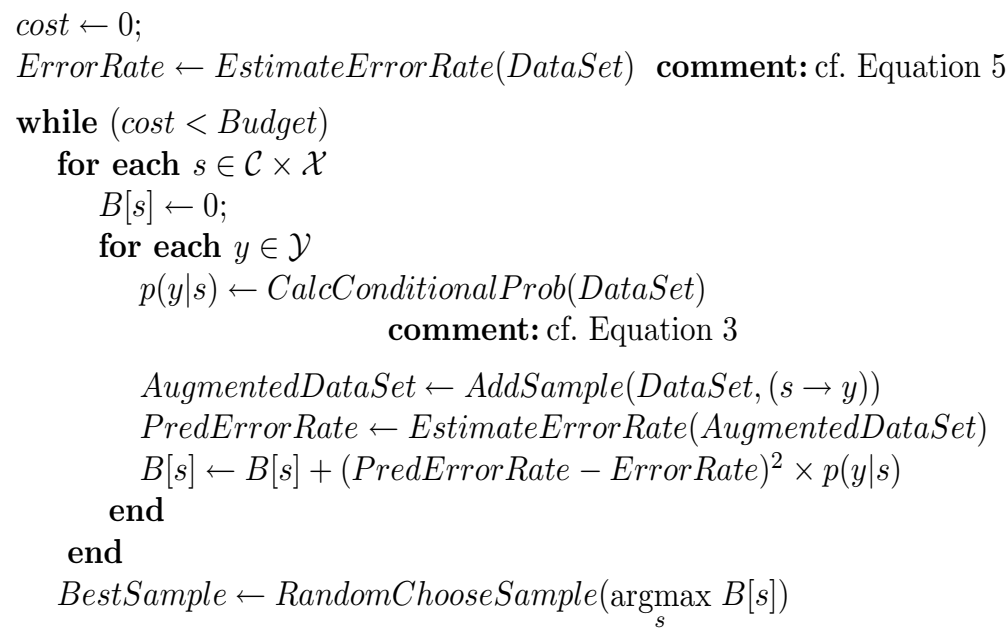

comment: Randomly select an incomplete sample among samples with max Benefit

DataSet $\leftarrow$ AddSample $($ DataSet,$($ BestSample $\rightarrow \operatorname{Extract} Y($ BestSample $)))$

comment: Measure $\mathbf{y}$ on BestSample and update DataSet

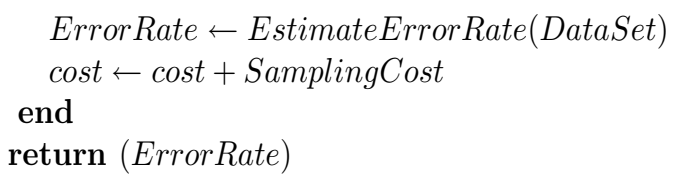

\section{Dataset for Experimentation}

To provide evidence that our method is effective in reducing costs in the biomedical domain we experimentally evaluated our method on a breast cancer Tissue Microarray dataset Although the biomarker evaluation problem is not relevant for this particular dataset we use it to demonstrate the utility of our approach.

The dataset was acquired using the recently developed technique of Tissue Microarray $[8]$ that improves the in-situ experimentation process by enabling the placement of hundreds of samples on the same glass slide. Core tissue biopsies are carefully selected in morphologically representative areas of original samples and then arrayed into a new "recipient" paraffin block, in an ordered array allowing for high-throughput in situ experiments. A TMA block contains up to 600 hundred tissue biopsies. TMA approach has a dramatic advantage over the conventional approach in performing in situ experiments on standard glass slides by allowing the simultaneous staining of hundreds of tissue samples from as many patients, ensuring experimental standardization and conserving the

${ }^{4}$ The data used for experimentation was collected by the Department of Histopathology and the Division of Medical Oncology, St. Chiara Hospital, Trento, Italy. Tissue Microarray experiments were conducted at the Department of Histopathology [2]. 
limited tissue resource, which is vital given the increasing number of candidate genes that need to be explored.

For each patient there is a record that described by clinical, histological and biomarkers information. The entire dataset consisted of 400 records defined by 11 features. Each of the clinical features is described by a binary status value and a time value. Some of the records have missing values. The data are described by the following features:

\section{Clinical Features}

1. the status of the patient (binary, dead/alive) after a certain amount of time (in months, integer from 1 to 160 )

2. the presence/absence of tumor relapse (binary value) after a certain amount of time (in months, integer from 1 to 160 months)

\section{Histological Features}

3. diagnosis of tumor type made by pathologists (nominal, 14 values)

4. pathologist's evaluation of metastatic lymphonodes (integer valued)

5. pathologist's evaluation of morphology (called grading, ordinal, 4 values)

Biomarkers Features

(manually measured by experts in TMA)

6. Percentage of nuclei expressing ER (estrogen receptor) marker.

7. Percentage of nuclei expressing PGR (progesterone receptor) marker.

8. Score value (combination of colour intensity and percentage of stained area measurements) of P53 (tumor suppressor protein) maker in cells nuclei.

9. Score value (combination of colour intensity and percentage of stained area measurements) of cerbB marker in cells membrane.

The learning task defined on this dataset is the prediction of the status of the patient (dead/alive or relapse) given some previous knowledge (histological information or known biomarkers). The goal is to choose the new biomarker which can be used along with the histological features that provides accurate prediction. The experiments address the issue of learning which additional feature has to be sampled.

The dataset was preprocessed as follows. Continuous features have been discretized to reduce the level of detail and to narrow the configuration space for the sampling problem. Feature values have been discretized encoded into binary variables according to the convention suggested by experts in the domain.

We designed 10 experiments corresponding to different learning situations. The experiments differ in the choice of attribute for the class label (c), the attributes used as the previous features $(\mathbf{x})$ and the feature used as the new candidate feature $(\mathbf{y})$. The various configurations are shown below. 


\begin{tabular}{rcccc}
\multicolumn{3}{c}{ Class Label (C) } & Known Features (X) & New Feature (Y) Size (\#) \\
\hline I & dead/alive & all histological information & PGR & 160 \\
II & dead/alive & all histological information & P53 & 164 \\
III & dead/alive & all histological information & ER & 152 \\
IV & dead/alive & all histological information & cerbB & 170 \\
V & relapse & all histological information & PGR & 157 \\
VI & relapse & all histological information & P53 & 161 \\
VII & relapse & all histological information & ER & 149 \\
VIII & relapse & all histological information & cerbB & 167 \\
IX & dead/alive & PGR, P53, ER & cerbB & 196 \\
X & relapse & PGR, P53, ER & cerbB & 198 \\
\hline
\end{tabular}

For the empirical evaluation we performed an additional preprocessing step of removing all the records with missing values for each experiment separately. For this reason the sizes of datasets used for different experiments are different.

\section{Experiments}

For each of the 10 experimental configurations described above, the random and MAC sampling schemes are compared for different number of acquired samples. The evaluation metric is computed as follows. For each choice of $\mathbf{x}$ and $\mathbf{y}$ we calculated the expected error rate $e_{F}$ of a maximum a posteriori classifier trained on the entire database (i.e., with all the values of $\mathbf{c}, \mathbf{x}$ and $\mathbf{y}$ known). Then for a given sample size $L$ we sampled $\mathbf{y}$ values on $L$ samples from the database (either by MAC or random sampling) and calculate the predicted error rate $e_{L}$ for each method. We then computed the root mean square difference between $e_{L}$ and $e_{F}$ over several runs of the sampling scheme. Under the assumption of unit cost for feature value acquisition the rms difference will measure the efficacy of a sampling scheme in estimating the error rate of a classifier trained on both $\mathbf{x}$ and $\mathbf{y}$ as a function of the number of feature values acquired.

To relate the evaluation measure used to the biological problem, we note that $e_{F}$ can be viewed as the true error rate of the classifier that uses the new biomarker $\mathbf{y}$ and $e_{L}$ as the estimate of the error rate after sampling $\mathbf{y}$ on $L$ samples. Since our goal is to predict the error rate accurately minimizing $L$, we can measure the effectiveness of our sampling algorithm by the rms difference between $e_{F}$ and $e_{L}$.

For each experiment we plotted the rms value against the number of samples probed which are shown in Figure 1, In each plot, to compare the MAC sampling scheme to the random method for cost effectiveness, we must compare the number of feature values sampled for a required rms error.

In some plots the rms value starts at zero before increasing to a maximum value. This happens in situations where the new feature $\mathbf{y}$ adds no new information for predicting the class $\mathbf{c}$ given the previous features $\mathbf{x}$. Therefore in the beginning of the sampling process, the estimated error rate is just the error rate obtained by using $\mathbf{x}$ which is the actual value. As more samples are added the 

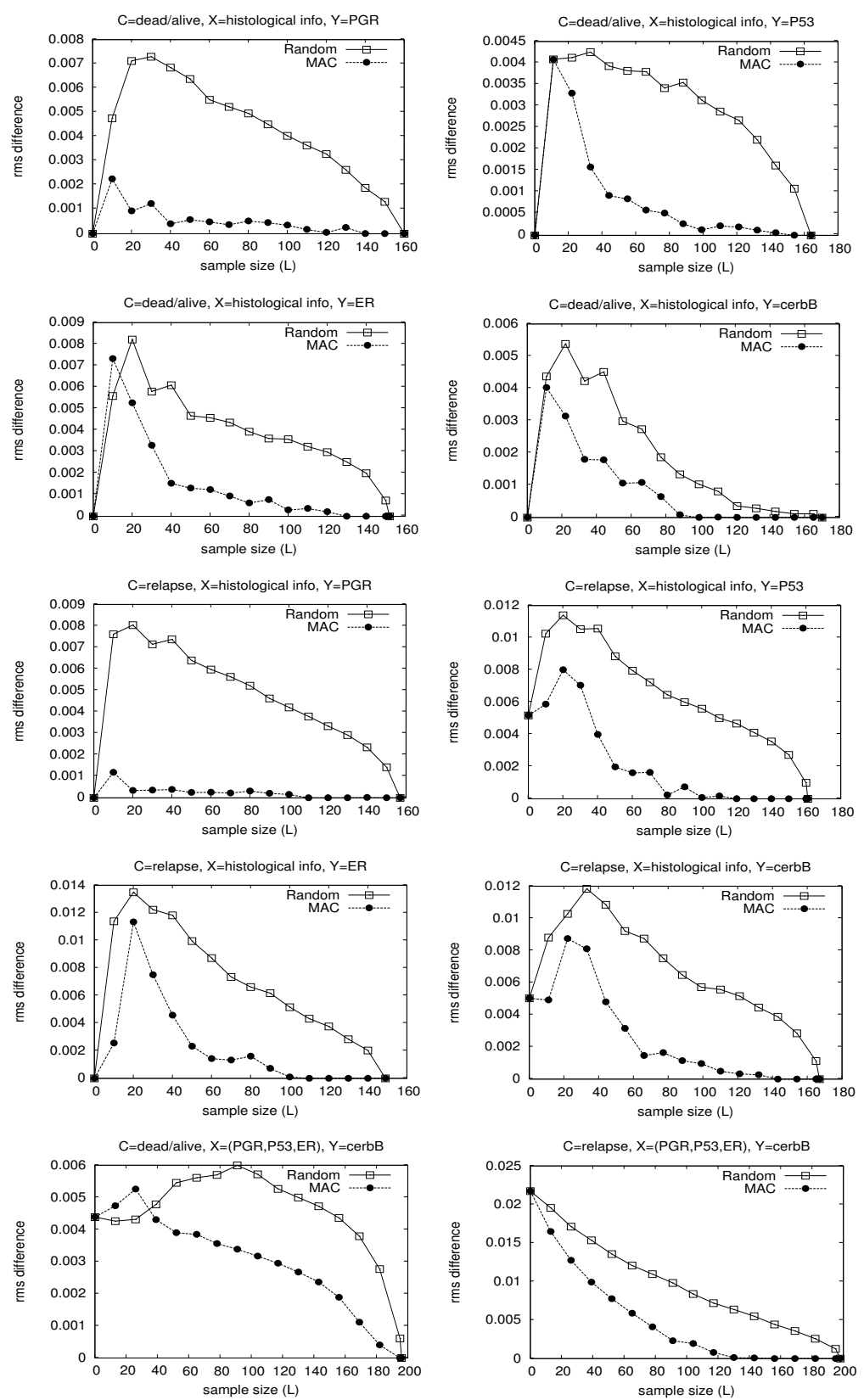

Fig. 1. Plots of the rms difference between the 'true' error rate of the classifier operating on the full feature space and that estimated from the acquired samples for all three sampling schemes as a function of the number of samples acquired. The features chosen for $\mathbf{c}, \mathbf{x}$ and $\mathbf{y}$ are also indicated. The rms value is computed from 500 runs of the sampling experiment for each configuration. The error bars are smaller than the markers used in the plots. 
estimate becomes optimistic predicting an error rate lower than the actual value until sufficiently large number of samples are added to learn the new feature is useless.

We observe from the plots that our MAC active sampling algorithm is significantly better in reducing the number of samples needed for error rate estimation than random sampling. For example in the top-left subplot, we observe that to obtain an error rate estimate with an rms error of 0.001 , MAC algorithm needs less than 40 samples as opposed to about 150 for random. This behaviour is observed in most of the experimental configurations.

In terms of the biomedical problem, this implies that using the MAC active sampling, we can evaluate a higher number of biomarkers on the same amount of bio-sample resource than using the standard random sampling method.

\section{Conclusions and Future Work}

We presented a preliminary solution to the problem of evaluating new biomarkers that enable better characterization of cancer while conserving the limited amount of well annotated biological samples. We showed experimentally that our active sampling algorithm holds promise in accurately evaluating the efficacy of the new biomarker with significantly fewer samples tested. This allows for the evaluation of more biomarkers using the biological samples at hand. One way to exploit the efficacy of the active sampling algorithm is to initially test the biomarkers on only a limited number of samples to discard uninformative biomarkers and proceed to test the remaining ones exhaustively.

There are still several open problems that need to be addressed. With the Tissue Microarray method several tissue samples are stained with the biomarker simultaneously. Therefore we need to extend our sampling algorithm by reasoning about incrementally sampling not one but a batch of samples. Although we can follow the same analysis presented above to derive an algorithm for the batch case, the algorithm would be computationally expensive. Therefore we need to make further approximations to derive a feasible algorithm. Since the Tissue Microarray process is complicated in the preparation of the slides, the costs involved are not uniform. We intend to develop active sampling algorithms for more general cost models. We also intend to investigate active sampling with other classification schemes. Our goal is to develop a fully functioning active sampling decision support system that can be employed for biomarker evaluation in biomolecular laboratories.

\section{References}

1. D.A.Cohn, Z.Ghahramani, and M.I.Jordan. Active learning with statistical models. In G. Tesauro, D. Touretzky, and T. Leen, editors, Advances in Neural Information Processing Systems, volume 7, pages 705-712. MIT Press, 1995.

2. F. Demichelis, A. Sboner, M. Barbareschi, and R. Dell'Anna. Tmaboost: an integrated system for comprehensive management of tissue microarray data. IEEE Trans. Inf. Technol. Biomed. In Press. 
3. D.J.C.MacKay. Information-based objective functions for active data selection. Neural Computation, 4(4):590-604, 1992.

4. D.Lizotte, O.Madani, and R.Greiner. Budgeted learning of naive-bayes classifiers. In Proceedings of the 19th Annual Conference on Uncertainty in Artificial Intelligence (UAI-03), pages 378-385, San Francisco, CA, 2003. Morgan Kaufmann.

5. T.R. Golub, D.K. Slonim, P. Tamayo, C. Huard, M. Gaasenbeek, J.P. Mesirov, H. Coller, M.L. Loh, J.R. Downing, M.A. Caligiuri, C.D. Bloomfield, and E.S. Lander. Molecular classification of cancer: class discovery and class prediction by gene expression monitoring. Science, 286(5439):531-537, 1999.

6. H.S.Seung, M.Opper, and H.Sompolinsky. Query by committee. In Proceedings of the fifth annual workshop on Computational learning theory, pages 287-294. ACM Press, 1992.

7. K.Chaloner and I.Verdinelli. Bayesian experimental design: A review. Statistical Science, 10:273-304, 1995.

8. J. Kononen, L.Bubendorf, A.Kallioniemi, M.Barlund, P.Schraml, S.Leighton, J.Torhorst, M.Mihatsch, G.Seuter, and O.P.Kallioniemi. Tissue microarrays for high-throughput molecular profiling of tumor specimens. Nature Medicine, 4(7):844-847, 1998.

9. M.Saar-Tsechansky and F.Provost. Active Sampling for Class Probability Estimation and Ranking. In Proc. Tth International Joint Conference on Artificial Intelligence, pages 911-920, 2001.

10. P.Sebastiani and H.P.Wynn. Maximum entropy sampling and optimal Bayesian experimental design. Journal of Royal Statistical Society, pages 145-157, 2000.

11. S.Tong and D.Koller. Support vector machine active learning with applications to text classification. In Proceedings of the International Conference on Machine Learning, pages 999-1006, 2000.

12. S.Veeramachaneni, E.Olivetti, and P.Avesani. Active feature sampling for low cost feature evaluation. Technical report, ITC-irst, 2005.

13. V.V.Fedorov. Theory of optimal experiments. Academic Press, New York, 1972.

14. Z.Zheng and B.Padmanabhan. On active learning for data acquisition. In Proceedings of the International Conference on Datamining, pages 562-570, 2002. 